\title{
Avaliação de Parametrizações de Camada Limite Planetária do Modelo WRF na Costa Norte do Nordeste do Brasil
}

\author{
Natanael Vieira de Sousa ${ }^{1}$ D, Adaiana Francisca Gomes da Silva ${ }^{2}$, Gilberto Ferno Fisch ${ }^{3}$, \\ João Bosco Verçosa Leal Junior ${ }^{1}$, Roberto Fernando da Fonseca Lyra ${ }^{4}$, \\ Emerson Mariano da Silva ${ }^{1}$ \\ ${ }^{1}$ Departamento de Física, Centro de Tecnologia, Universidade Estadual do Ceará, Fortaleza, CE, \\ Brasil. \\ ${ }^{2}$ Instituto Tecnológico de Aeronáutica, Divisão de Energia, São José dos Campos, SP, Brasil. \\ ${ }^{3}$ Instituto de Aeronáutica e Espaço, São José dos Campos, SP, Brasil. \\ ${ }^{4}$ Universidade Federal de Alagoas, Instituto de Ciências Atmosféricas, Maceió, AL, Brasil.
}

Recebido em: 16 de Novembro de 2017 - Aceito em: 17 de Março de 2018

\begin{abstract}
Resumo
Este trabalho tem como objetivo avaliar duas simulações do modelo WRF que diferem em sua parametrização da Camada Limite Planetária (CLP). Foram utilizados dados de 72 radiossondas lançadas em Fortaleza (CE) entre 5 e 25 de Abril de 2011 nos horários sinóticos. A altura da CLP foi a variável avaliada e os valores observados, calculados pelo método do gradiente vertical da temperatura potencial, foram de $245 \pm 28 \mathrm{~m}$ para o período noturno e de $925 \pm 63 \mathrm{~m}$ para o período diurno. As parametrizações de CLP avaliadas foram Asymmetric Convective Model V2 e Mellor Yamada Nakanish and Niino 2.5. O desempenho destas foi avaliado por comparação estatística com dados observados, utilizando-se o Erro Médio Absoluto (EMA) e o índice refinado de concordância de Willmott $\left(\mathrm{d}_{\mathrm{r}}\right)$. Para a ACM2 obtiveramse valores de EMA iguais a $282 \mathrm{~m}$ e $519 \mathrm{~m} \mathrm{e} \mathrm{d}_{\mathrm{r}}$ de 0,53 e 0,37 , respectivamente, para os períodos noturno e diurno. Com MYNN2,5, foram encontrados valores de EMA entre $312 \mathrm{~m}$ e $266 \mathrm{~m}$, com $\mathrm{d}_{\mathrm{r}}$ entre 0,59 e 0,56 para os períodos noturno ediurno, respectivamente. A parametrização MYNN2,5 mostrou-se mais eficiente, considerando ambas as métricas. No entanto, somente para 00UTC, a ACM2 apresentou melhor desempenho.
\end{abstract}

Palavras-chave: modelagem atmosférica, radiossonda, altura da CLP, ventos alísios.

\section{Evaluation of the Planetary Boundary Layer Parameterizations of WRF Model in North Northeast Coast at Brazil}

\begin{abstract}
This work aims to evaluate two simulations of the WRF model that differ in their parameterization of the Planetary Boundary Layer (PBL). For this goal, we have used data from 72 radiosondes launched in Fortaleza (CE) between April 5 th to 25 th of 2011 in the synoptic times. The PBL height was the evaluated metric and the observational data were calculated through the vertical potential temperature method, obtaining $245 \pm 28 \mathrm{~m}$ for nighttime and $925 \pm 63 \mathrm{~m}$ for daytime. The Asymmetric Convective Model V2 (ACM2) and the Mellor Yamada Nakanish and Niino 2.5 (MYNN2.5) PBL parameterizations have been evaluated. The skills of these parameterizations were evaluated by statistical comparison with observational data through Mean Absolute Error (MAE) and the refined Willmott's index of agreement $\left(\mathrm{d}_{\mathrm{r}}\right)$. For ACM2, MAE was $282 \mathrm{~m}$ and $519 \mathrm{~m}$ and $\mathrm{d}_{\mathrm{r}}$ was 0.53 and 0.37 for the nighttime and daytime periods, respectively. In the MYNN2.5 simulation, MAE values were found between 312 and $266 \mathrm{~m}$, with $\mathrm{d}_{\mathrm{r}}$ between 0.59 and 0.56 for the nighttime and daytime, respectively. At 06, 12 and 18 UTC, MYNN2.5 was better, considering both metrics. However, ACM2 presented better performance only at 00 UTC.
\end{abstract}

Keywords: atmospheric modeling, radiosonde, planetary boundary layer, trade winds.

Autor de Correspondência: Natanael Vieira de Sousa, natanaelfisico@gmail.com. 


\section{Introdução}

Em sistemas de tempo e clima, os processos físicos dentro de nuvens ainda não são totalmente compreendidos. A partir desse fato, foi desenvolvido um projeto de pesquisa, denominado Projeto Chuva (significado da sigla Chuva em Português: Processos de nuvem associados aos principais Sistemas Precipitantes no Brasil: Uma Contribuição à modelagem da escala de nuvens), cujo objetivo científico foi o de melhorar parametrizações físicas em modelos de mesoescala.

Os quatro principais objetivos do Projeto Chuva (Portal Chuva) foram: I) melhorar a previsão de tempo em alta resolução; II) criar uma climatologia dos processos físicos nas nuvens dos principais regimes de precipitação do Brasil; III) desenvolver ferramentas para a previsão imediata de tempestades; IV) desenvolver a componente solo no satélite brasileiro GPM. Este projeto foi realizado através de campanhas experimentais em algumas cidades de diferentes regiões do país.

A segunda cidade onde houve campanha experimental foi em Fortaleza, Estado do Ceará, para representar as características atmosféricas da costa Norte da região Nordeste do Brasil. Os dados observados do projeto Chuva (descrito em detalhes em Machado et al. 2014) foram obtidos através das radiossondas realizadas em Fortaleza-CE e tiveram como objetivo avaliar a dinâmica da camada limite local. Os dados simulados foram utilizados como uma forma alternativa de representar resultados de características do ciclo diário da CLP na costa Norte-Nordeste do Brasil, em comparação/validação com dados extraídos da radiossonda, visando propor combinações adequadas de parametrizações da região durante um mês (abril) da estação chuvosa (considerando que o período de estação chuvosa na região é de fevereiro a maio (Brabo Alves et al., 2000).

Resultados de pesquisas sobre Camada Limite Planetária (CLP) são importantes para estudos de clima e de qualidade do ar (Seidel et al., 2010), assim como para modelar as trocas entre a superfície e a atmosfera, de quantidade de movimento, energia, umidade, carbono e poluentes (Mcgrath-Spangler e Molod, 2015). Segundo Stull (1988) o ciclo diário da CLP é altamente variável. Foken (2008) apresenta a seguinte abordagem acerca deste ciclo: após o nascer do sol (período diurno), a atmosfera é aquecida pelo fluxo de calor turbulento da superfície do solo, e a camada de inversão formada durante a noite é desfeita (processo de erosão da Camada Limite Noturna). A seguir, ocorre o desenvolvimento da camada de mistura rapidamente, com o crescimento da Camada Limite Convectiva (CLC). Esta camada é muito turbulenta, possui uma extensa camada de mistura (tipicamente entre 1000$2000 \mathrm{~m}$ ), sendo delimitada em sua parte superior por uma zona de entranhamento. Um pouco antes do pôr do sol, com a inversão do fluxo de calor sensível, a camada limite estável se desenvolve a partir do solo, alcançando uma profundidade variável entre 100-200 m.

Algumas parametrizações de CLP são mais adequadas, por exemplo, para simular as condições de tempo para pousos e decolagens de aeronaves (Powers et al., 2003; Comin et al., 2016) e outras podem ser utilizadas para levantamento de potencial eólico (Krogsaeter e Reuder, 2014). Assim, bons resultados de estudos da CLP podem melhorar previsões de velocidade dos ventos, nevoeiros e surgimento de nebulosidade.

Os estudos da CLP em regiões próximas às regiões tropicais têm impactos em vários setores, bem como no setor aeroespacial, uma vez que muitos centros de lançamentos de foguetes e veículos aeroespaciais localizam-se ao redor destas latitudes. No Brasil, particularmente, destaca-se a costa Norte da região Nordeste. Com o objetivo de estudar a altura da CLP em regiões tropicais, Fisch (2012) apresenta alguns resultados observacionais, usando o método do perfil vertical da temperatura potencial calculados por radiossonda e radiômetro de microondas.

Ainda em específico para a região tropical, Leal Junior et al. (2013) avaliaram a performance de 6 esquemas de parametrização de camada limite planetária do modelo WRF, a saber: 1.Yonsei University [YSU], desenvolvida para camada limite estável; 2. Mellor-Yamada-Janjic [MYJ]; 3. Asymmetric Convective Model version 2 [ACM2]; 4. Quasi Normal Scale Elimination [QNSE]; 5.Mellor-Yamada-NakanishiNiino Level 2.5 (MYNN2.5) e 6. Bougeault-Lacarrere [BouLac]. Os autores chegaram à conclusão de que as opções ACM2 e MYNN2.5 apresentaram os melhores resultados para a reprodução dos campos de velocidade e direção do vento à superfície. Também Silva e Fisch (2014) avaliaram o modelo WRF para a previsão do vento no Centro de Lançamento de Alcântara (CLA) no Maranhão (Nordeste do Brasil) tendo como um dos principais objetivos determinar a melhor parametrização de CLP para esta localidade, mas que também pudesse ser utilizada em toda a costa norte da região NE.

Carvalho et al. (2014) afirmam, através de resultados obtidos a partir de várias métricas estatísticas (bias, raiz do erro quadrático médio, desvio padrão e coeficiente de correlação), que a parametrização ACM2 com o esquema de Pleim Xiu na superfície mostrou globalmente as melhores pontuações estatísticas comparado às características observadas dos dados de vento para seis regiões da Península Ibérica.

Outros modelos de mesoescala, tal como o BRAMS (Brazilian Regional Atmospheric Modeling System), foi usado por Rodrigues et al. (2011) para um período de condições meteorológicas extremas, neste caso uma chuva intensa provocada por um sistema meteorológico chamado "onda leste", que causou grandes inundações em RecifePE (Nordeste do Brasil). Um segundo modelo regional, o 
MM5, foi usado por Pereira Neto e Oyama (2011) para o CLA com o objetivo de melhorar o esquema de parametrização convectiva Kain-Fritsch (KF2) da região.

O presente trabalho tem como objetivo avaliar duas parametrizações de camada limite planetária do modelo WRF (versão 3.6), a saber: ACM2 e MYNN2.5, para a região da costa norte do NE do Brasil. As métricas estatísticas utilizadas foram o Erro Médio Absoluto (EMA) e o índice refinado de concordância de Willmott $\left(\mathrm{d}_{\mathrm{r}}\right)$ (Willmott, Robeson e Matsura, 2014).

\section{Materiais e Metodos}

\subsection{Projeto Chuva e campanha experimental de Fortaleza}

A Campanha de Fortaleza do Projeto Chuva ocorreu entre 01 e 30 de Abril de 2011, com o apoio local das seguintes instituições: Fundação Cearense de Meteorologia e Recursos Hídricos (FUNCEME); Defesa Civil do Estado do Ceará (DCECE) e Defesa Civil da cidade de Fortaleza (DCMF).

A pesquisa apresentada neste artigo foi desenvolvida após a obtenção de resultados das atividades do grupo de trabalho 4 (Características da camada limite planetária para diferentes processos de nuvens e forma de precipitação pluviométrica), responsável pela caracterização da CLP.

\subsection{Condições de tempo durante a campanha de Fortaleza do projeto Chuva}

De acordo com boletins meteorológicos da campanha Fortaleza, entre os dias 6 e 8 de Abril havia pouca nebulosidade no Estado do Ceará, causada pela presença de um vórtice ciclônico de altos níveis (VCAN), tornando a precipitação muito baixa. Em 12 de Abril (ver Fig. 1), existiam sistemas convectivos na maior parte do estado, principalmente perto da costa, devido ao posicionamento mais ao sul da Zona de Convergência Intertropical (ZCIT), que é um dos principais sistemas meteorológicos causadores de chuvas sobre o Ceará (Ferreira e Mello, 2005).

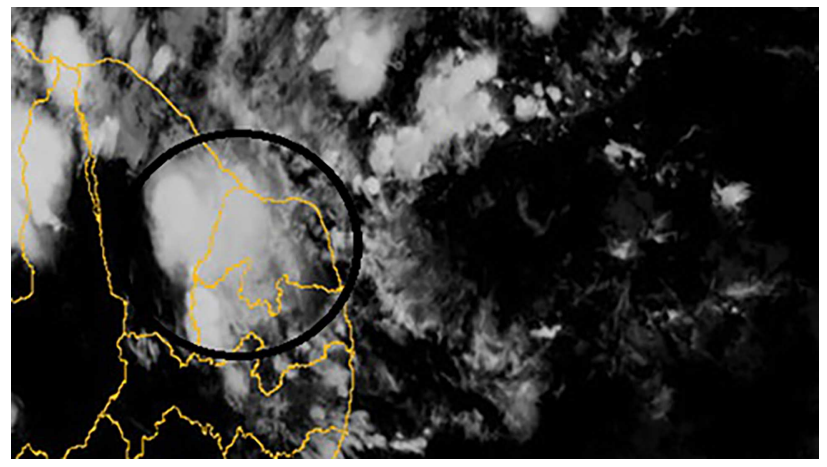

Figura 1 - Imagem do Sistema Meteorológico atuante na Costa Norte do Nordeste do Brasil em 12 de Abril de 2011 às 12 UTC. Fonte: Satélite METEOSAT - FUNCEME (2015).
Em 13 de Abril (Fig. 2), a ZCIT deslocou-se para o Nordeste do Ceará, produzindo chuvas significativas nesta parte do estado. Nos três dias consequentes (14, 15 e 16 de Abril), havia nebulosidade apenas na cidade de Fortaleza e sua região metropolitana (Fig. 3).

A Fig. 4 mostra a ZCIT mais próxima do Nordeste do Estado causando chuvas na maior parte do estado em 19 de Abril de 2011, ao passo que a Fig. 5 apresenta a ZCIT na linha do Equador, cobrindo todo o Estado do Ceará com nebulosidade. Estes sistemas atmosféricos foram aqueles que estiveram presentes ao longo do período estudado.

\subsection{Dados Observacionais: radiossondagem e pluviometria}

Os dados observacionais utilizados (perfis de vento, temperatura e umidade relativa do ar, dentre outros) foram coletados por radiossondas (modelo Vaisala RS92G) em Fortaleza, no período entre 05 e 25 de Abril de 2011 (coincidindo com o período da estação chuvosa da região) nos horários das $00,06,12$ e 18 UTC, totalizando 72 lançamentos. Este modelo de radiossonda possui as seguintes

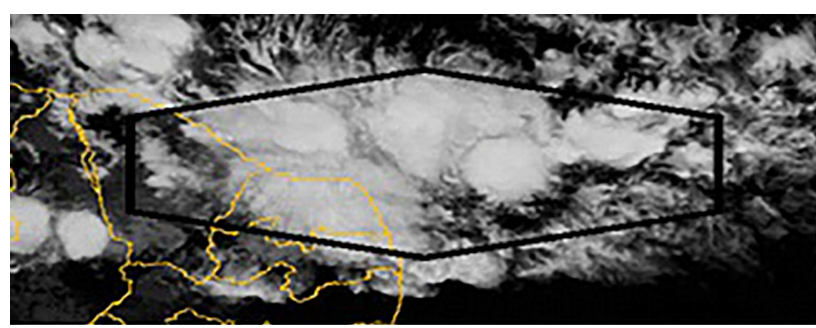

Figura 2 - Idem Figura 1, porém para o dia 13 de Abril.

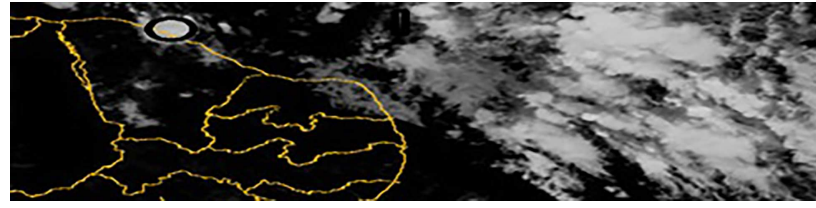

Figura 3 - Idem Figura 1, mas para o dia 16 de Abril.

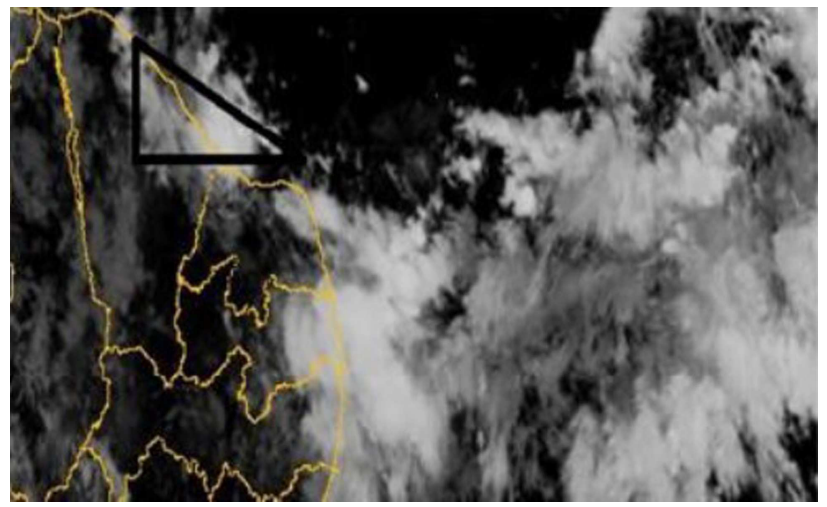

Figura 4 - Idem Figura 1, mas para o dia 19 de Abril. 


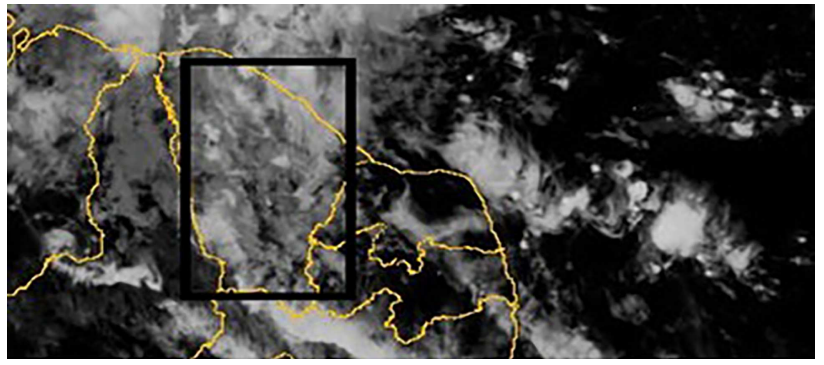

Figura 5. - Idem Figura 1, mas para o dia 25 de Abril.

especificações técnicas: sensor de umidade com capacitor de película fina, sensor de pressão de silicone, sensor de temperatura do ar (termopar) que possui proteção contra resfriamento evaporativo e as medições do vento (calculados pelo sistema GPS), com banda de transmissão de dados em $403 \mathrm{MHz}$. O local de lançamento, situado a aproximadamente $12 \mathrm{~km}$ da costa, foi a estação do Instituto Nacional de Meteorologia (INMET) em Fortaleza, identificada pelo número sinótico 81758 segundo a Organização Meteorológica Mundial (OMM) e localizada a $3,75^{\circ}$ de latitude Sul e $38,54^{\circ}$ de longitude Oeste.

Para determinar a altura da camada limite planetária, foi utilizado o método do gradiente vertical da temperatura potencial, identificando a altura da inversão térmica de separação entre CLP e a atmosfera livre, como descrito em Seidel et al. (2010).

Durante o período de estudo, os dados diários de precipitação acumulada foram obtidos a partir de um pluviômetro instalado no sítio PICI da FUNCEME, localizado a uma distância de $11 \mathrm{~km}$ da estação do INMET. A Fig. 6 apresenta a série temporal da chuva observada durante a campanha experimental.

\subsection{Discussões físicas das parametrizações da camada limite convectiva (CLC)}

\subsubsection{Asymmetric Convective Model V2 (parametrização CLC - SIM1)}

A parametrização ACM2 consiste em uma combinação de fechamento local e não local. Assim utiliza-se uma transição matricial para fluxos não-locais. A técnica chave para os dois esquemas é o desenvolvimento de acordo com a altura, ou seja, o topo da camada mais baixa do modelo e a porção da razão de mistura entre os dois esquemas (locais e não locais) resultam de tal forma como se estivessem processados sozinhos, como se o esquema local fosse processado separadamente do não-local, porém ao mesmo tempo, e o resultado fosse baseado nesta duplicidade de processamento. Esta parametrização é mais indicada para grandezas como umidade, temperatura, vento, gases traço e razão de mistura.

A formulação matemática Eq. (1) que descreve esta parametrização é:

$$
\begin{aligned}
& \frac{\partial C_{i}}{\partial t}=\mathbf{f}_{\text {conv }} M_{u} C_{1}-\mathbf{f}_{\text {conv }} M_{d_{\mathrm{i}}} C_{i}+ \\
& \mathbf{f}_{\text {conv }} \mathbf{M}_{\mathbf{d}_{\mathrm{i}}+\mathbf{1}} C_{\boldsymbol{i}+\mathbf{1}} \frac{\Delta_{z_{i+1}}}{\Delta_{z_{i}}}+\frac{\partial}{\partial_{z}}\left[K_{c}\left(1-\mathbf{f}_{\text {conv }}\right) \frac{\partial C_{\boldsymbol{i}}}{\partial z}\right]
\end{aligned}
$$

onde $M u$ é a razão de mistura ascendente, $M d_{i}$ é a razão de mistura descendente da i-ésima camada em relação à anterior, $C_{1}$ representa o escalar na camada mais baixa do modelo e $\Delta z i$ é a espessura da i-ésima camada. $\mathrm{f}_{\text {conv }}$ (Eq. (2)) é o parâmetro chave para controlar a contribuição das misturas local e não-local. Uma parte de $\mathrm{f}_{\text {conv }}$ é derivada da razão do fluxo de calor não local em relação ao fluxo de calor total no topo da camada superficial e segue abaixo a descrição de $\mathrm{f}_{\text {conv }}$ :

$$
\mathbf{f}_{\text {conv }}=1 /\left[1+\frac{k^{-\frac{2}{3}}}{0,1 a}\left(-\frac{\boldsymbol{h}}{\boldsymbol{L}}\right)^{-\frac{1}{3}}\right]
$$

onde $a$ é uma constante com valor 7,2, $k$ é a constante de von Karman cujo valor é $0,4, h$ é a altura da CLP e $L$ a escala de comprimento de Monin-Obukhov.

No primeiro membro da Eq. (1), o primeiro termo diz respeito aos fluxos ascendentes não locais na superfície. O segundo termo indica a compensação do fluxo descendente à camada imediatamente mais baixa. $\mathrm{O}$ terceiro termo significa o fluxo descendente assimétrico de

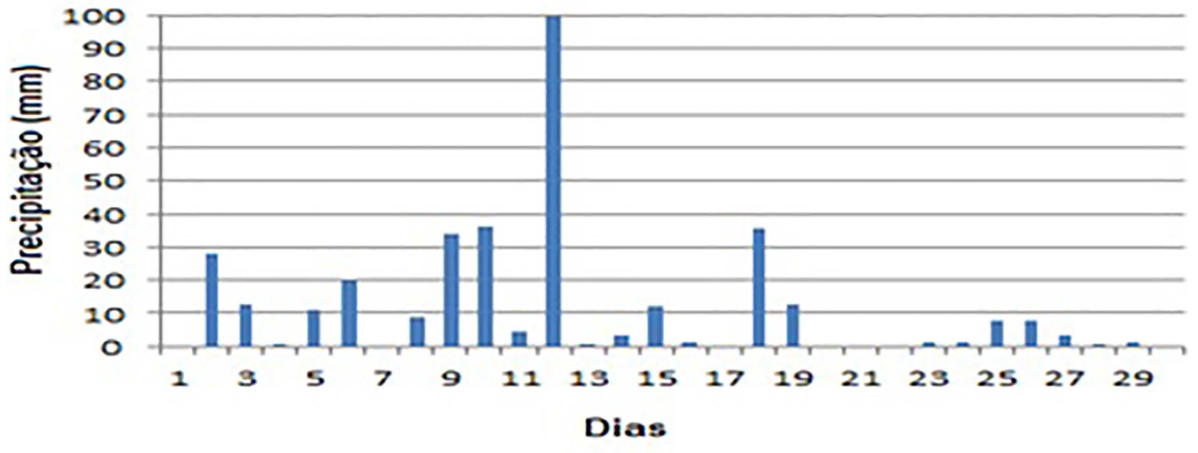

Figura 6 - Precipitação pluviométrica acumulada diária observada durante a campanha de Fortaleza do projeto Chuva. 
uma camada imediatamente mais alta. O último termo infere a difusão turbulenta ascendente local (Pleim, 2007a; 2007b).

A troca de massa não local promovida pela ACM2 é uma representação física de transporte ascendente devido ao deslocamento convectivo que se aplicam a qualquer quantidade. A parametrização ACM2 foi elaborada com a proposta de tornar mais realista tanto a modelagem meteorológica como a química da atmosfera.

\subsubsection{Mellor Yamada Nakanish and Niino 2.5 (parametrização CLC - SIM2)}

A parametrização Mellor Yamada Nakanish and Niino usa resultados de Large-Eddy-Simulations (LES) e produz maior Energia Cinética Turbulenta (ECT) e comprimento de mistura, o que leva a suaves aumentos nas profundidades das camadas de mistura (Kim et al., 2013). Nakanish e Niino (2006) afirmam que esta parametrização é oriunda da Mellor Yamada só que com nível 2,5; com este fim vários pesquisadores fizeram inclusões e modificações envolvendo velocidade, gradiente de temperatura, ECT e comprimento de escala.

\subsubsection{Razão da escolha de ACM2 e MYNN2.5 e confronto de vantagens e desvantagens}

Silva (2013) mostrou que as parametrizações ACM2 e MYNN2.5 são mais indicadas para a região próxima à linha do equador; sendo que MYNN2.5 apresentou maior destaque entre ACM2, YSU e MYN.

As principais vantagens da ACM2 são: esquema de fechamento de primeira ordem; possui ambas as componentes local e não-local; combina componentes tanto de escala maior (super) e menor (sub) do que a grade do modelo; ao adicionar uma componente de difusão de vórtices ao transporte não local representa melhor o vento pois os fluxos de massa são definidos explicitamente; adequado para transporte consistente de $\mathbf{u}, \mathbf{v}$ e $\Theta$ (Silva, 2013). As desvantagens mais acentuadas de ACM2 são: o modelo de difusão de vórtices resulta num valor mais alto da altura da CLP; durante a estação chuvosa pode gerar uma cobertura nebulosa (Silva, 2013).

As vantagens em maior destaque da MYNN2.5 são: esquema de fechamento de nível 2.5 baseado na energia cinética turbulenta local; menor custo computacional; estabilidade numérica; corrige o conteúdo de água líquida; corrige o crescimento da camada limite (Silva, 2013). Suas principais desvantagens são: reproduz a condensação na CLP considerando temperatura potencial da água líquida $\left(\theta_{l}\right)$ e conteúdo total de água liquida $\left(q_{w}\right)$; bom principalmente em situações de mudança de fase na CLP; as variâncias de $\left\langle\theta_{l}{ }^{2}\right\rangle,\left\langle\theta_{l} q_{w}\right\rangle$ e $\left\langle q_{w}{ }^{2}\right\rangle$ são determinados diagnosticamente, negligenciando a tendência temporal e os termos de difusão (Silva, 2013).

\subsection{Configuração das simulações}

A configuração completa utilizada nas simulações realizadas com o modelo WRF é mostrada na Tabela 1, sendo que o mapa do domínio utilizado é apresentado na Fig. 7. Os domínios aninhados 1, 2 e 3 possuem áreas iguais a $271,8 \mathrm{~km}^{2} ; 532,4 \mathrm{~km}^{2}$ e $1944 \mathrm{~km}^{2}$, respectivamente. Além de análises para cada instante, também foram realizadas avaliações estatísticas para os horários das radiossondagens [horários sinóticos, representando o período diurno (sondagens das 12 e 18 UTC) e noturno (sondagens das 00 e 06 UTC)].

\subsection{Avaliação estatística das parametrizações da Camada Limite Planetária}

Os resultados das simulações de CLP foram comparados com dados observacionais obtidos por radiossonda e analisados através do EMA (Eq. (3)), do dr Eqs. (4)-(5) e do teste $t$ student com nível de significância $(\alpha)$ de $5 \%$

Tabela 1 - Configuração do Modelo WRF.

\begin{tabular}{|c|c|}
\hline Modelo & Configurações \\
\hline Dados de análise & FNL \\
\hline Espaçamento de grade horizontal (km) & 16,$2 ; 5,4 ; 1,8$ \\
\hline Dimensão horizontal (km) & $1944 ; 532,4 ; 271,8$ \\
\hline Níveis verticais & 45 \\
\hline Topo do modelo (hPa) & 10 \\
\hline \multirow[t]{2}{*}{ Parametrização de radiação } & Longa: RRTM (MLAWER et al., 1997) \\
\hline & Curta: Dudhia (DUDHIA, 1989) \\
\hline Parametrização de Microfísica & WSM6 (HONG \& LIM, 2006) \\
\hline Parametrização de Convecção & Kain-Fritsch (KAIM, 2004) \\
\hline \multirow[t]{2}{*}{ Parametrização de CLP para turbulência } & SIM1: ACM2 (PLEIM, 2007) \\
\hline & SIM2: MYNN2.5 (NAKANISHI e NIINO, 2006) \\
\hline \multirow[t]{2}{*}{ Parametrização de CLP solo-superfície } & SIM1: Pleim-Xiu (Pleim e Gilliam, 2009) \\
\hline & SIM2: NOAH (NIU et al., 2011) \\
\hline
\end{tabular}




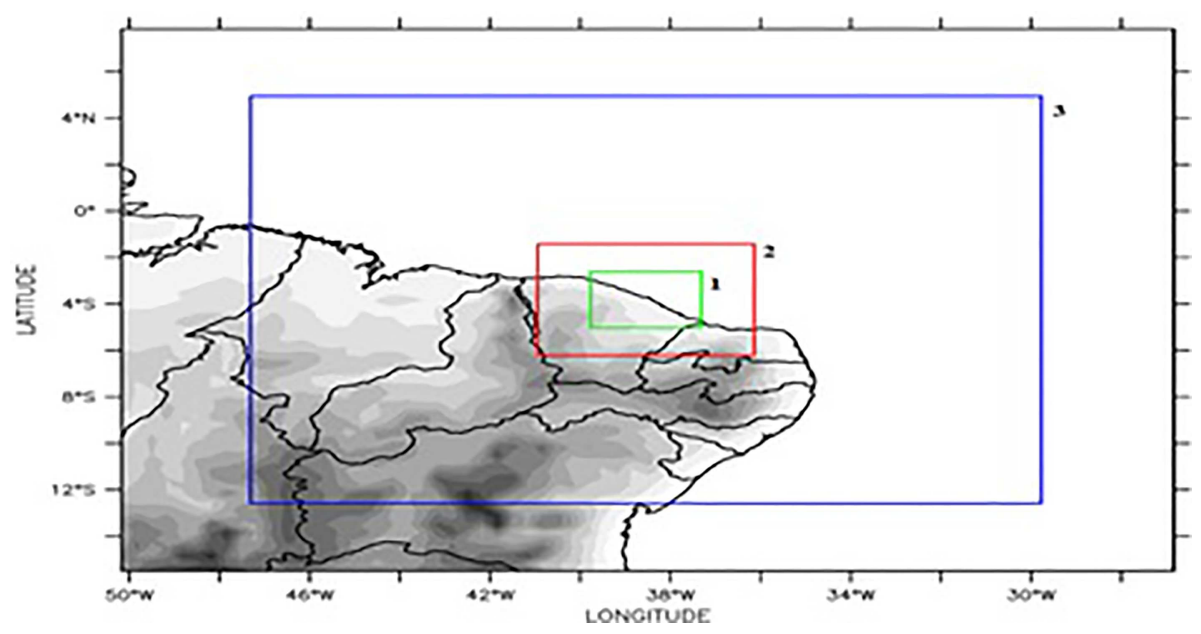

Figura 7 - Mapa de domínio usado em ambas as simulações.

Eq. (6).

$$
\begin{gathered}
\operatorname{EMA}=\frac{1}{\mathrm{~N}} \sum_{\mathrm{i}=1}^{\mathrm{N}}\left|\mathrm{S}_{\mathrm{i}}-\mathrm{O}_{\mathrm{i}}\right| \\
\mathrm{d}_{\mathrm{r}=}=\left\{1-\mathrm{c} \frac{\sum_{\mathrm{i}=1}^{\mathrm{N}}\left|\mathrm{O}_{\mathrm{i}}-\overline{\mathrm{O}}\right|}{\sum_{\mathrm{i}=1}^{\mathrm{N}}\left|\mathrm{P}_{\mathrm{i}-\mathrm{O}_{\mathrm{i}}}\right|}\right\} \\
\operatorname{para} \sum_{\mathrm{i}=1}^{\mathrm{N}}\left|\mathrm{P}_{\mathrm{i}}-\mathrm{O}_{\mathrm{i}}\right| \leq \mathrm{c} \sum_{\mathrm{i}=1}^{\mathrm{N}}\left|\mathrm{O}_{\mathrm{i}}-\overline{\mathrm{O}}\right| \\
\mathrm{d}_{\mathrm{r}=}=\left\{\mathrm{c} \frac{\sum_{\mathrm{i}=1}^{\mathrm{N}}\left|\mathrm{O}_{\mathrm{i}}-\overline{\mathrm{O}}\right|}{\sum_{\mathrm{i}=1}^{\mathrm{N}}\left|\mathrm{P}_{\mathrm{i}-\mathrm{O}_{\mathrm{i}}}\right|}-1\right\} \\
\sum_{\mathrm{i}=1}^{\mathrm{N}}\left|\mathrm{P}_{\mathrm{i}}-\mathrm{O}_{\mathrm{i}}\right|>\mathrm{c} \sum_{\mathrm{i}=1}^{\mathrm{N}}\left|\mathrm{O}_{\mathrm{i}}-\overline{\mathrm{O}}\right| \\
\mathrm{z}=\frac{\overline{\mathbf{X}}-\mu}{\sigma / \sqrt{\mathrm{N}}}
\end{gathered}
$$

onde EMA é a diferença absoluta entre o valor simulado $\left(\mathrm{S}_{\mathrm{i}}\right)$ e o observado $\left(\mathrm{O}_{\mathrm{i}}\right)$ a cada instante, e $\mathrm{d}_{\mathrm{r}}$ avalia quanto o valor simulado se aproxima do observado. A métrica $d_{r}$ varia de -1 a 1 , sendo que o valor "1" significa concordância perfeita. $\mathrm{O}$ termo $c$ é uma constante relativa à frequência de saída do modelo, à qual foi atribuído o valor 2 (Wilmott et al., 2012). A variável $\mathrm{P}_{\mathrm{i}}$ corresponde ao valor simulado ("previsto") para o instante (i), sendo que $\mathrm{O}_{\mathrm{i}}$ indica o valor observado no mesmo instante $\mathrm{i}$ e $\overline{\mathrm{O}}$ representa a média dos valores observados. $\overline{\mathbf{X}}$ é a média amostral, $\mu$ é a média populacional, $\sigma$ é o desvio padrão populacional e $\mathrm{N}$ o tamanho da amostra para então ser possível encontrar o escore $\mathrm{z}$ que possui um correspondente $\alpha$.

\section{Resultados e Discussões}

\subsection{Resultados observacionais e perfis verticais da altura da camada limite planetária}

A Tabela 2 mostra os valores de altura de CLP obtidos

\begin{tabular}{|c|c|c|c|c|}
\hline Dia & 00 UTC (m) & $06 \mathrm{UTC}(\mathrm{m})$ & $12 \mathrm{UTC}(\mathrm{m})$ & $18 \mathrm{UTC}(\mathrm{m})$ \\
\hline 5 & 420 & - & 686 & 860 \\
\hline 6 & 462 & 127 & 675 & 975 \\
\hline 7 & 341 & 235 & 827 & 756 \\
\hline 8 & 243 & 247 & 1273 & 632 \\
\hline 9 & - & - & - & 964 \\
\hline 10 & 66 & 199 & 947 & 1327 \\
\hline 11 & 349 & 272 & 725 & 773 \\
\hline 12 & 253 & 328 & 823 & 1520 \\
\hline 13 & & 142 & 684 & 753 \\
\hline 14 & 168 & - & - & 808 \\
\hline 15 & - & 382 & 541 & 800 \\
\hline 16 & 284 & 117 & 430 & 587 \\
\hline 17 & 182 & 119 & 950 & 1318 \\
\hline 18 & 292 & 263 & 845 & 1112 \\
\hline 19 & 171 & 314 & 760 & 1292 \\
\hline 20 & 127 & - & - & - \\
\hline 21 & - & - & - & 808 \\
\hline 22 & 142 & 322 & 672 & 604 \\
\hline 23 & 299 & 451 & 475 & 643 \\
\hline 24 & 134 & 153 & - & 1055 \\
\hline 25 & - & 528 & 600 & - \\
\hline Média & 245 & 262 & 744 & 925 \\
\hline Desvio padrão & 112 & 121 & 206 & 276 \\
\hline
\end{tabular}
pelo método do gradiente vertical da temperatura potencial, calculados a partir dos dados de radiossondas. $\mathrm{O}$ valor da maior altura para o horário das 00 UTC foi $462 \mathrm{~m}$ em 6 de

Tabela 2 - Alturas observacionais da CLP (em metros) obtidas pelas radiossondas em Fortaleza. 

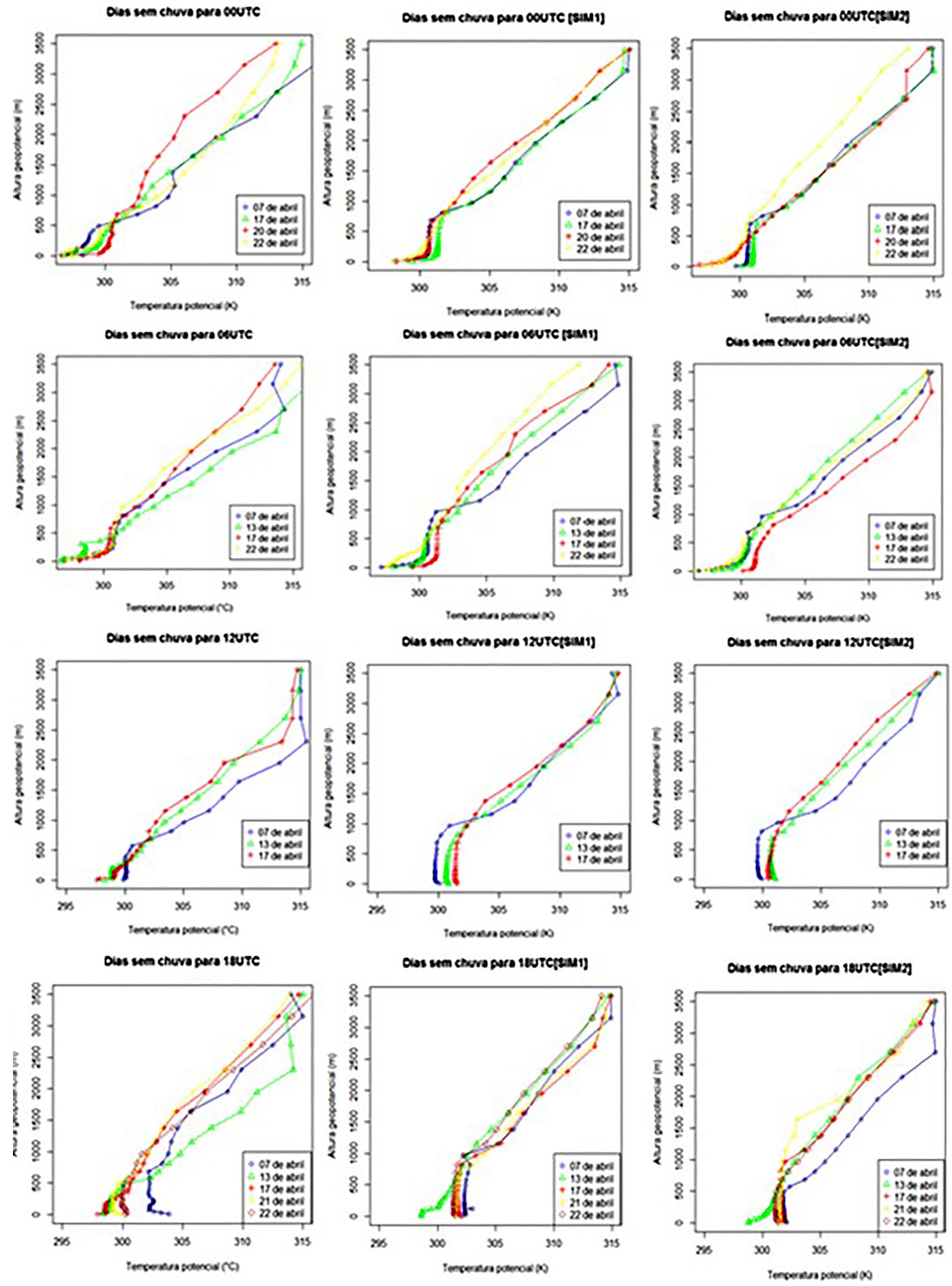

Figura 8 - Perfis verticais de temperatura potencial para dias não chuvosos. Os quadros da esquerda, centrais e da direita mostram, respectivamente, perfis observados e simulados SIM1 e SIM2. Cada horário (00, 06, 12 e 18 UTC) é apresentado separadamente em (a), (b), (c) e (d), respectivamente. Os dias representados encontram-se descritos na legenda de cada quadro. 
abril, o menor valor foi em 10 de Abril com $66 \mathrm{~m}$, e o valor médio foi $245 \mathrm{~m}$. Em $06 \mathrm{UTC}$, foram obtidos $528 \mathrm{~m}$ ( $25 \mathrm{de}$ Abril) e $117 \mathrm{~m}$ (16 de Abril) para os valores mais altos e mais baixos respectivamente. A média em 06 UTC ficou em $262 \mathrm{~m}$. O valor médio de altura da CLP para as 12 UTC foi $744 \mathrm{~m}$ e para as $18 \mathrm{UTC}, 925 \mathrm{~m}$. O valor mais alto em 12 UTC foi $1273 \mathrm{~m}$ em 8 de Abril e o mais baixo foi em 16 de Abril com $430 \mathrm{~m}$. Os extremos mínimo e máximo da altura da CLP observados ao longo de todo o período analisado estão destacados em negrito na tabela. Os espaços com (-) representam lançamentos sem dados válidos ou que não houve lançamentos. Sousa (2014) faz uma análise detalhada da evolução destes valores e sua associação com sistemas atmosféricos que ocorreram durante o experimento de Fortaleza. As Figs. 8a, b, c e d apresentam os valores da temperatura potencial versus altura geopotencial observadas e simuladas por SIM1 e SIM2 nos horários 00, 06, 12 e 18 UTC para dias sem chuva. É possível observar a comparação entre os resultados nos diferentes dias em cada nível de altura para os primeiros 28 níveis verticais $(10,32,53$, $75,97,118,140,162,184,208,236,269,310,359,418$, $490,578,685,814,970,1157,1380,1642,1948,2300$,
2701, 3151 e 3500 m). A Fig. 9 mostra os perfis verticais de temperatura potencial, nos mesmos níveis, para um dia antes de um evento chuvoso, seguido de um chuvoso e um pós-evento chuvoso para verificação do comportamento da CLP no horário 00 UTC (noturno) e 18 UTC (diurno) nos painéis superior e inferior, respectivamente. De maneira análoga, são também apresentados os resultados para a razão de mistura do vapor d'água nas Figs. 10 (a, b, c e d) e Fig. 11.

Nas Figs. 8a e 10a, referentes a 00UTC para dias sem chuva, percebe-se que SIM1 (ACM2) teve melhor desempenho do que SIM2 (MYNN2.5) tendo como valores de referência os obtidos pelas radiossondas tanto para temperatura potencial como para razão de mistura do vapor d'água. A justificativa física para tal resultado consiste: a) temperatura potencial - no fato da parametrização ACM2 ser recomendada para representar transporte de vento zonal, vento meridional e temperatura potencial. No entanto, gerou uma altura da CLP um pouco maior no referido horário para os dias apontados; b) razão de mistura - o resultado é derivado da duplicidade de

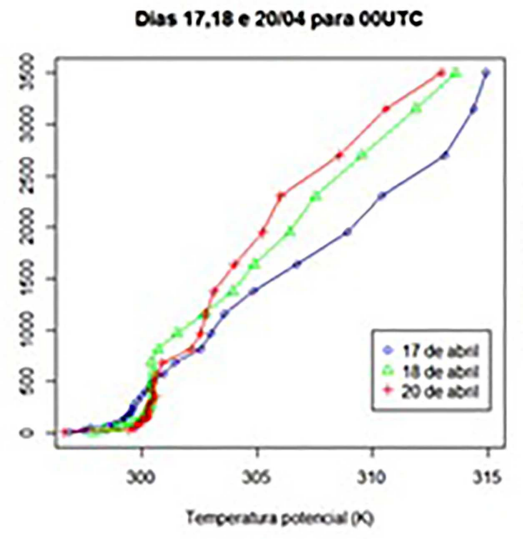

Dias 17,:8 e 21/0s para 186TC

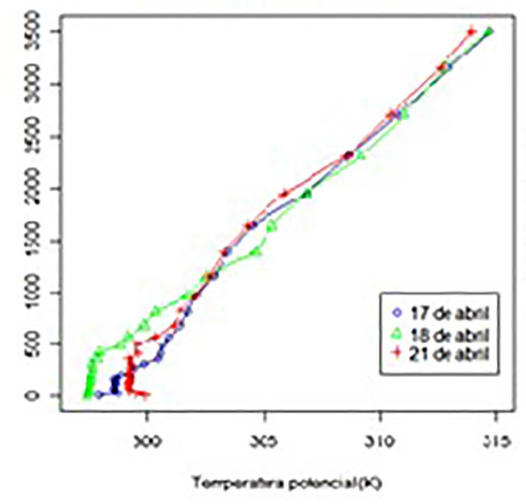

Dios 17.18 e $2010 \mathrm{~s}$ pora o0uTC[stasi]

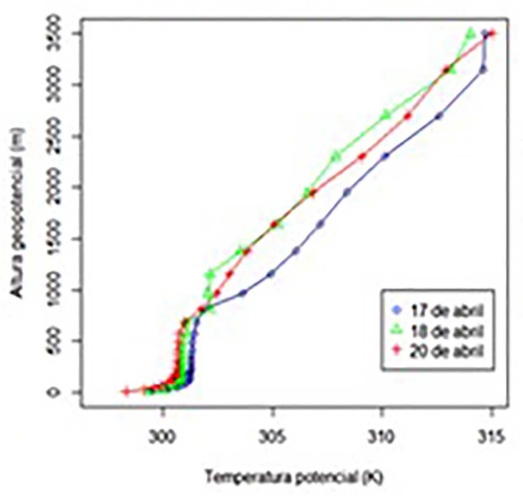

Olas 17.18 e 21/0s para 18UTC[SImai]

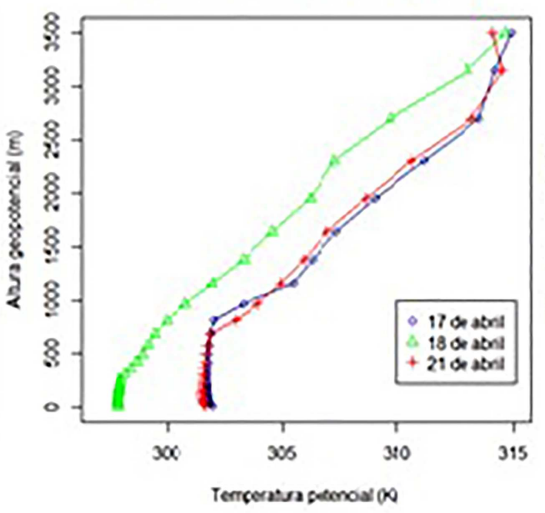

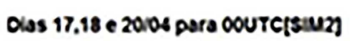

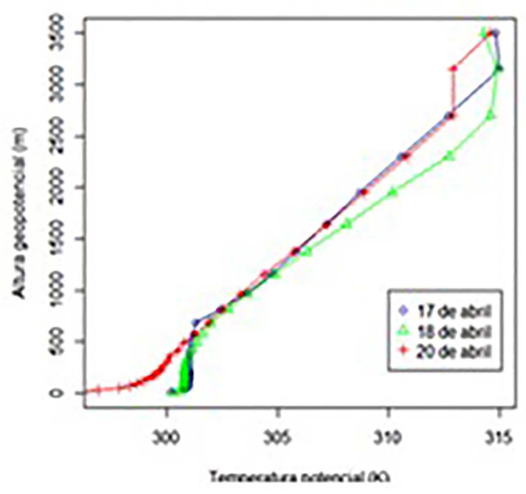

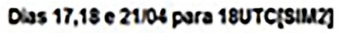

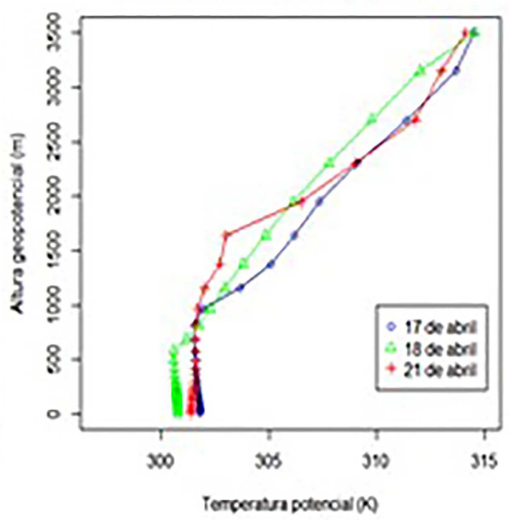

Figura 9 - Perfis verticais de temperatura potencial para um dia pré-chuva (17 de abril), chuvoso (18 de abril) e pós-chuvoso (20 ou 21 de abril) às 00 UTC (quadros superiores) e 18 UTC (quadros inferiores). Os quadros da esquerda, centrais e da direita mostram, respectivamente, perfis observados e simulados SIM1 e SIM2. 

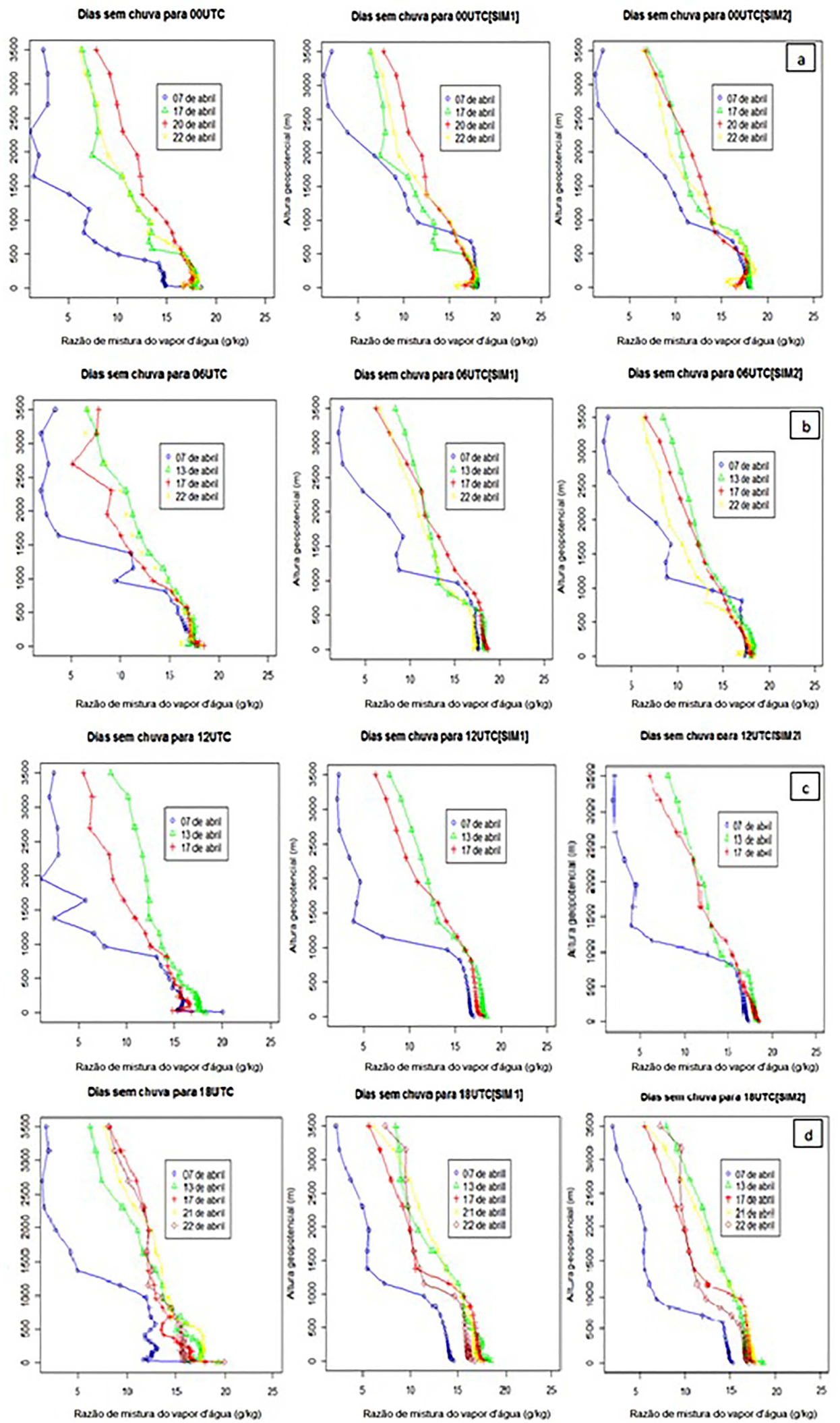

Figura 10 - Perfis verticais de razão de mistura do vapor d'água para dias não chuvosos. Os quadros da esquerda, centrais e da direita mostram, respectivamente, perfis observados e simulados SIM1 e SIM2. Cada horário (00, 06, 12 e 18 UTC) é apresentado separadamente em (a), (b), (c) e (d), respectivamente. Os dias representados encontram-se descritos na legenda de cada quadro. 

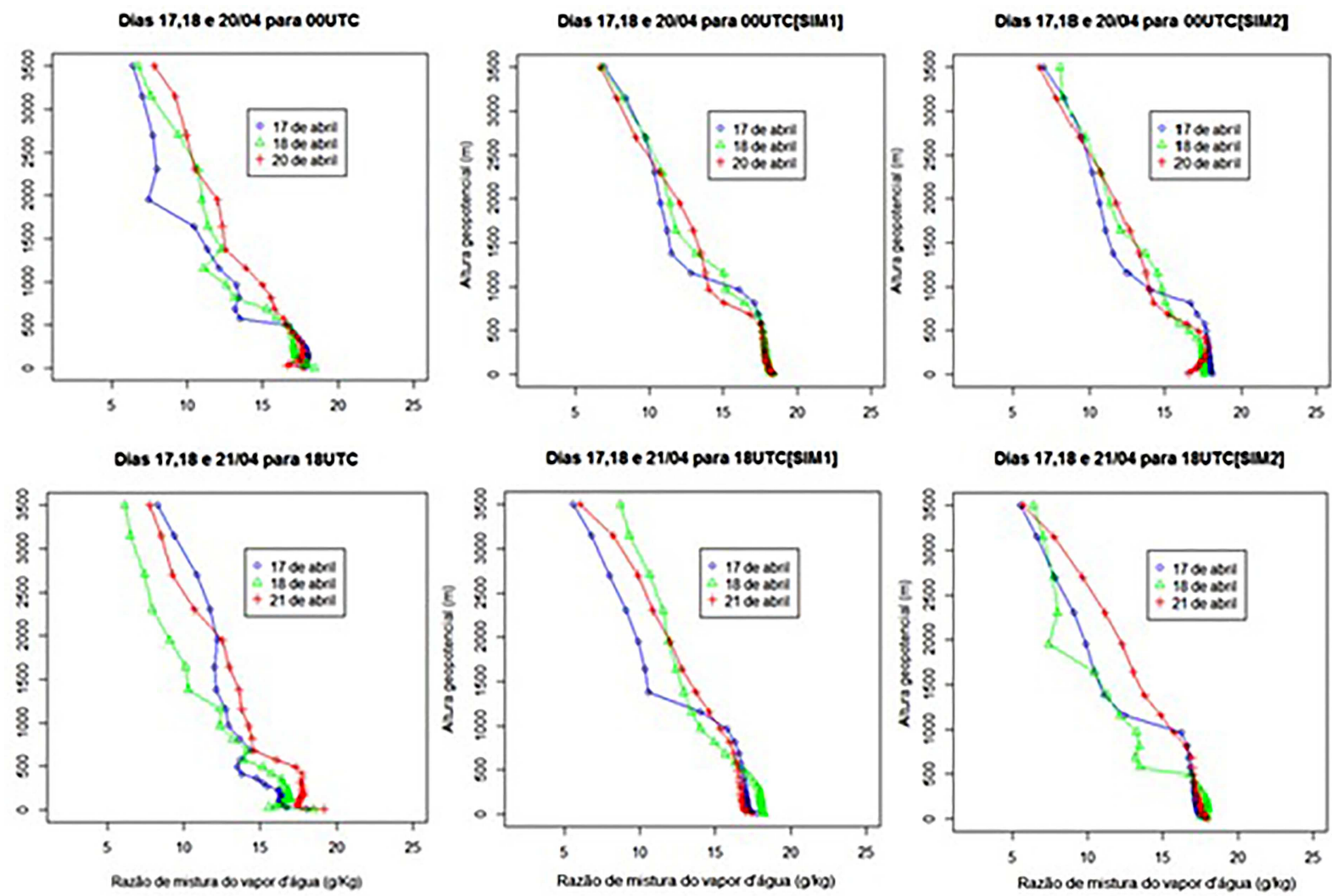

Figura 11 - Perfis verticais de razão de mistura do vapor d'água para um dia pré-chuva (17 de abril), chuvoso (18 de abril) e pós-chuvoso (20 ou 21 de abril) às 00 UTC (quadros superiores) e 18 UTC (quadros inferiores). Os quadros da esquerda, centrais e da direita mostram, respectivamente, perfis observados e simulados SIM1 e SIM2.

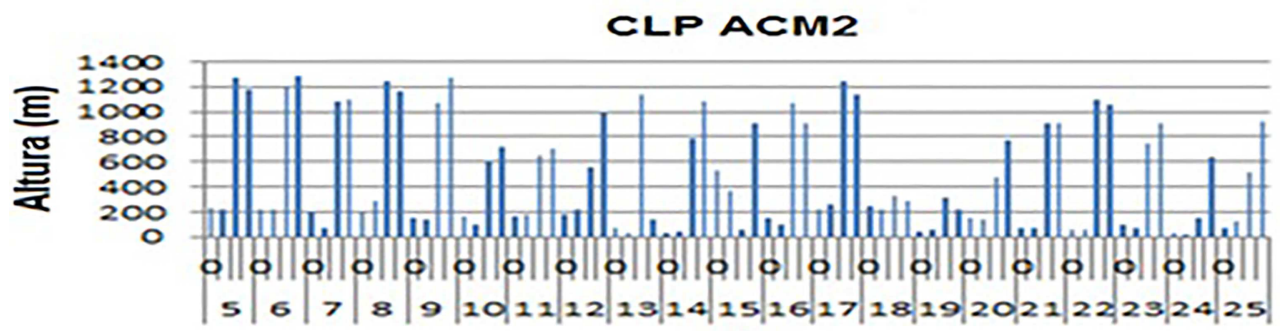

Dias

Figura 12. - Série temporal da altura da Camada Limite Planetária obtida pela SIM1 (parametrização ACM2).

CLP MYNN2,5

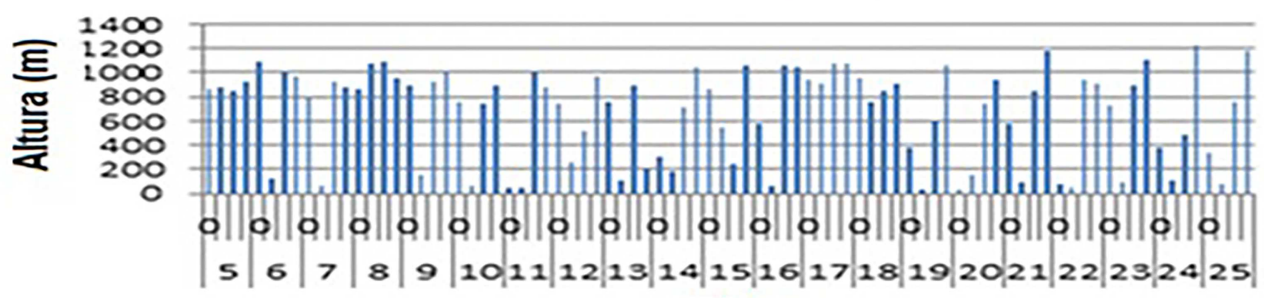

Dias

Figura 13. - Série temporal da altura da Camada Limite Planetária obtida pela SIM2 (parametrização MYNN2,5). 
processamento local e não-local, fazendo um combinado entre a razão de mistura ascendente e descendente em sua formulação matemática.

As Figs. 8b e 10b não apontam uma melhor parametrização considerando temperatura potencial e razão de mistura do vapor d'água.

A Fig. 10c apresentou resultados não satisfatórios para o dia 17 para a representação da razão de mistura em ambas as parametrizações. Contudo, os resultados das duas opções de turbulência foram parecidos com os resultados de radiossondagens para os demais dias com MYNN.2.5 sendo um pouco melhor, pois esta parametrização corrige o crescimento de água liquida.

Não foi possível obter resultados que apontassem a melhor parametrização de acordo com as Figs. 8d e 10d, pois os resultados de SIM1 e SIM2 para as duas variáveis meteorológicas apresentaram resultados díspares em relação ao observado.

Nos quadros superiores das Fig. 9 e 11, percebe-se um melhor desempenho de SIM1 na representação dos dias pré-chuvoso e chuvoso. Tal resultado é devido ao fato do período de estudo ser a estação chuvosa e nesta época esta parametrização de turbulência favorece o crescimento de cobertura nebulosa. Nos resultados dos quadros inferiores das Figs. 9 e 11, foi possível identificar a parametrização MYNN2.5 como a mais indicada para o período diurno (18 UTC), esta representou bem os três dias (pré-chuvoso; chuvoso e pós-chuvoso) devido à esta parametrização produzir uma maior energia cinética turbulenta.

\subsection{Resultados das simulações numéricas}

A Fig. 12 mostra os valores da altura da CLP às 00 , 06, 12 e 18 UTC através da simulação 1 (SIM1), que utiliza a parametrização ACM2. O dia com maior valor foi 06 de Abril em 18 UTC, com 1279 m. 24 de Abril foi o dia com mais baixo valor às 06 UTC, $20 \mathrm{~m}$. As alturas variaram de aproximadamente $100-200 \mathrm{~m}$, no período noturno, para valores entre $1100-1279 \mathrm{~m}$ no período diurno, mostrando claramente um ciclo diário da CLP.

A Fig. 13 mostra os valores de altura da CLP às 00 , 06, 12 e 18 UTC através da simulação 2 (SIM2), que utilizou a parametrização MYNN2.5. O intervalo entre as alturas máximas foi de $200 \mathrm{~m}$. Os valores mais baixos foram encontrados geralmente às 00 e 06 UTC, sendo a altura de $42 \mathrm{~m}$ a mais baixa de todas, obtido às 06 UTC do dia 20 de Abril. $\mathrm{O}$ valor da CLP mais alto foi de $1213 \mathrm{~m}$ às 18 UTC do dia 24 de Abril. De modo geral, estas estimativas mostraram valores mais homogêneos ao longo do mês, embora com alturas máximas menores (valores entre 900$1100 \mathrm{~m})$.

A Tabela 3 apresenta em resumo os resultados das métricas estatísticas consideradas para as duas simulações, separadas por horário e por simulação. Os índices "_1" e " 2" remetem respectivamente a SIM1 e SIM2. Com estes dados, é possível obter os seguintes resultados: a parametrização ACM2 foi melhor às 00 UTC, com EMA_2 atingindo um valor maior que o dobro do valor de EMA_1. No horário 06 UTC, foi possível obter resultados estatísticos conclusivos que apontaram SIM2 como melhor para simular a altura da CLP em ambas as métricas estatísticas. Em 12 e 18 UTC, a parametrização SIM2 também foi melhor, reforçando MYNN2,5 como a melhor opção de parametrização de turbulência no início da manhã e tarde (hora local) que representam a CLP diurna.

\section{Conclusões}

Os resultados obtidos com a parametrização ACM2 representaram satisfatoriamente a CLP em dias não chuvosos, com resultados próximos aos observados no horário 00 UTC. Ainda no período noturno, em dias secos seguintes aos dias chuvosos, a CLP foi mais alta, o que indica que estes parâmetros, de acordo com as estatísticas, apontaram ACM2 eficiente para representar a CLP noturna na cidade de Fortaleza; do ponto de vista da análise de significância foram obtidos: $\alpha \_S 1$ xobs_noturno $=$ 0,0029 e $\alpha \_$S2xobs_noturno $=0,0070$; embora ambos os resultados sejam satisfatórios em nível de 5\% de significância, a ACM2 apresentou desempenho um pouco maior que o dobro da eficácia. Porém, nos horários 06, 12 e 18 UTC (período diurno), SIM2 apresentou resultados melhores para a reprodução da altura da CLP em relação ao mesmo período simulado com a SIM1. Às 18 UTC, por exemplo, obteve-se EMA igual a $269 \mathrm{~m}$ para MYNN2.5, contra $718 \mathrm{~m}$ para ACM2. Em todos os períodos analisados $\left(00,06,12,18\right.$ UTC, diurno e noturno) a métrica $\mathrm{d}_{\mathrm{r}_{-}} 2$

Tabela 3 - Análise dos resultados de ACM2 (SIM1) e MYNN2,5 (SIM2) usando o Erro Médio Absoluto e o Índice de Concordância de Willmott usado em ambas as simulações.

\begin{tabular}{lcccc}
\hline Hora & EMA_1 $(\mathrm{m})$ & EMA_2 $(\mathrm{m})$ & $\mathrm{d}_{\mathrm{r}_{-}} 1$ & \\
\hline 00UTC & 139 & 357 & 0,48 & \\
06UTC & 425 & 268 & 0,23 & 0,50 \\
12UTC & 320 & 264 & 0,64 & 0,45 \\
18UTC & 718 & 269 & 0,51 & 0,69 \\
Noturno & 282 & 312 & 0,37 & 0,64 \\
Diurno & 519 & 266 & 0,53 & 0,59 \\
\hline
\end{tabular}


apresentou melhores resultados que a $d_{r_{-}} 1$, os resultados dos testes de significância [para o conjunto 00, 06, 12 e 18 UTC] mostraram $\alpha \_S 1 x o b s \_24 h=0,0500$ e $\alpha \_$S2xobs_24h $=0,0110$. Com esses resultados, é possível sugerir que MYNN2.5 é a parametrização mais indicada do que ACM2 para regiões tropicais, principalmente para o ciclo diário completo. Contudo, o teste de significância apontou: $\alpha \_S 2 x o b s \_d i u r n o=0,2470$ e $\alpha$ S 1 xobs_diurno $=$ 0,5390 , ou seja, muito maiores que 0,05 impossibilitando uma conclus yo a partir desta métrica. A conclusão a partir das métricas $\mathrm{d}_{\mathrm{r}_{-}}$e EMA concorda com Silva e Fisch (2014), que fizeram esta avaliação considerando o regime de ventos na região da costa litorânea do Maranhão.

\section{Agradecimentos}

Os autores agradecem à FAPESP pela disponibilidade de dados do projeto Chuva (processo ${ }^{\circ} 2009$ /15235-8) e à FUNCAP pelo apoio financeiro via bolsa de mestrado ao primeiro autor.

\section{Referências}

BRABO ALVES, J.M.; XAVIER, T.M.B.S.; FERREIRA, A.G.; MELLO, N.G.S.; Verificação de Prognósticos Sazonais de Precipitação no Estado do Ceará utilizando a técnica dos quantis. Revista Brasileira de Meteorologia, v. 15, n. 2, p. 73-85, 2000.

CARVALHO, D.; ROCHA, A.; GOMEZ-GESTEIRA, M.; SILVA SANTOS, C. Sensitivity of the WRF model wind simulation and wind energy production estimates to planetary boundary layer parameterizations for onshore and offshore areas in the Iberian Peninsula. Applied Energy, v. 135, n. 135, p. 234-246, 2014.

COMIN, A.N.; SOUZA, R.B.; ACEVEDO, O.C.; ANABOR,V. nálise do Desempenho do Modelo Weather Research and Forecasting (WRF) com Diferentes Esquemas de Microfísica e Camada Limite Planetária na Ilha Deception, Antártica. Revista Brasileira de Meteorologia, v. 31, n. 4, p. 415-427. 2016.

DUDHIA, J. Numerical study of convection observed during the winter monsoon experiment using a mesoescale two dimensional model. Journal of the Atmospheric Science, v. 46, n. 20, p. 3077-3107, 1989.

FERREIRA, A.G.; MELLO, N.G.S. Principais Sistemas atmosféricos atuantes sobre a região Nordeste do Brasil e a influencia dos oceanos Pacífico e Atlântico no clima da região. Revista Brasileira de Climatologia, v. 1, n. 1, p. 15-28, 2005.

FISCH, G. The Heights of the Atmospheric Boundary Layer at a Coastal Region use remote sensing and in situ measurements. In:16th International Symposium for the Advanced Boundary Layer Remote Sensing. Boulder: Mathematics in University of Colorado. 2012.

FOKEN, T. Micrometeorology. 3ed. Berlin, Heidelberg: Springer. 2008.
HONG, S.; LIM, J.J. The WRF Single Moment 6-class Microphysics scheme (WSM6). Journal of the Korean Meteorological Society, v. 42, n. 2, p. 129-151, 2006.

KAIM, J.S. The Kain-Fritsch Convective Parameterization: An Update. Journal of Applied Meteorology, v. 43, n. 1, p. 170-181, 2004.

KIM, Y.; SARTELET, K.; RAUT, J.; CHAZETTE, P. Evaluation of the Weather Research and Forecast/Urban Model Over Greater Paris. Boundary Layer Meteorology, v. 149, n. 1, p. 105-132, 2013.

LEAL JUNIOR, J.B.V.; PELLEGRINI, C.C.; ALMEIDA, G.P.; FISCH, G.; LYRA, R.F.F.; DA SILVA JUNIOR, R.S. Avaliando parametrizações de Camada Limite Planetária do WRF utilizando dados de velocidade e direção do vento do projeto Chuva em Fortaleza. Ciência e Natura, Edição Esp. Dez., p. 236-238, 2013.

MACHADO, L.A.T.; SILVA DIAS, M.A.F.; MORALES, C.; FISCH, G.; VILA, D.; ALBRECHT, R.; GOODMAN, S.J.; et al. The CHUVA Project - how does convection vary across Brazil? Bulletin of the American Meteorological Society, v. 95, n. 9, p. 1365-1380, 2014.

MCGRATH-SPLANGER, E.L.; MOLOD, A. Comparison of GEOS-5 AGCM Planetary Boundary Layer depths computed with various definitions. Atmospehric Chemistry and Physics, v. 14, n. 13, p. 6717-6727, 2014

MLAWER, E.J.; TAUBMAN, S.J., BROWN, P.D.; IACONO, M.J.; CLOUGH, S.A. Radiative transfer for inhomogeneous atmospheres: RRTM, a validated correlated-k model for the longwave. Journal of Geophysical Research, v. 102, n. D14, p. 16,663-16,682, 1997.

NAKANISH, M.; NIINO, H. An Improved Mellor-Yamada Level-3 Model: Its Numerical Stability and Aplicattion to a Regional Prediction of Advection Fog. Boundary Layer Meteorology, v. 119, n. 2, p. 397-407, 2006.

NIU, G.; YANG, Z.; MITCHELL, K.E.; CHEN, F.; EK, M.B.; BARLAGE, M.; KUMAR, A.; et al., The community Noah land surface model with multiparameterization options (Noah-MP): 1. Model description and evaluation with local scale measurements. Jornal of Geophysical Research, v. 116, D12109, p. 1-19, 2011.

PEREIRA NETO, A.V. ; OYAMA, M.D. Mudanças no Esquema de Convecção profunda Kain-Fritsch para a região do centro de lançamento de Alcântara. Revista Brasileira de Meteorologia, v. 26, n. 4, p.579-590, 2011.

PLEIM, J. A Combined Local and Nonlocal Closure Model for The Atmospheric Boundary Layer. Part I: Model Description and Testing. Journal of Applied Meteorology and Climatology, v. 46, Special issue: NOAA/EPA Golden Jubilee, p. 1383-1395, 2007.

PLEIM, J.E.; GILLIAM, R. An Indirect Data Assimilation Scheme for Deep Soil Temperature in the Pleim-Xiu Land Surface Model. Journal of Applied Meteorology and Climatology, v. 48, n. 7, p. 1362-1376, 2009.

POWERS, J.G.; MONAGHAN, A.J.; CAYETTE, A.M.; BROMWICH, D.H.; KUO, Y.-H.; MANNING, K.W. Realtime mesoscale modeling over Antarctica. American Meteorological Society, v. 84, n. 11, p. 1533-1545, 2003.

RODRIGUES, H.P.; SOUZA, E.P.; MARIANO, E.B. Análise da Influência de uma área urbanizada em variáveis 
meteorológicas através do uso do modelo BRAMS. Revista de Geografia, v. 28, n. 3, 2011.

SEIDEL, D.J.; AO, C.O.; LI, K. Estimating climatological planetary boundary layer heights from radiosonde observations: Comparison of methods and uncertainty analysis. Journal of Geophysical Research, v. 115, n. D16113, p. 1-15, 2010.

SILVA, A.F.G.; FISCH, G. Avaliação do Modelo WRF para a previsão do perfil do vento no centro de lançamento de Alcântara. Revista Brasileira de Meteorologia. v. 29, n. 2, p. $259-270,2014$

SILVA, A.F.G. Avaliação do modelo WRF ao perfil de vento no centro de lançamento de Alcântara. Dissertação de Mestrado em Meteorologia, Instituto Nacional de Pesquisas Espaciais. 76f. 2013

SOUSA, N.V. O modelo WRF no Projeto Chuva: Avaliação das Parametrizações de CLP durante o experimento de
Fortaleza. Mestrado em Ciências Físicas Aplicadas, Universidade Estadual do Ceará. 90f. 2014.

STULL, R.B. An Introduction to Boundary Layer Meteorology. 2 ed. Dordrecht: Kluwer, 1988.

WILLMOTT, C.J.; ROBESON, S.M.; MATSUURA, K. A refined index of model performance. International Journal of Climatology, v. 32, n. 13, p. 2088-2094, 2012.

\section{Endereços de Internet}

Portal Chuva: www.chuvaproject.cptec.inpe.br.

This is an Open Access article distributed under the terms of the Creative Commons Attribution License, which permits unrestricted use, distribution, and reproduction in any medium, provided the original work is properly cited. 